\title{
Semi-quantitative analyses of metabolic systems of human colon cancer metastatic xenografts in livers of superimmunodeficient NOG mice
}

\author{
Akiko Kubo Mitsuyo Ohmura $\cdot$ Masatoshi Wakui $\cdot$ Takahiro Harada \\ Shigeki Kajihara $\cdot$ Kiyoshi Ogawa $\cdot$ Hiroshi Suemizu $\cdot$ Masato Nakamura $•$ \\ Mitsutoshi Setou $\cdot$ Makoto Suematsu
}

Received: 29 November 2010 /Revised: 7 February 2011 / Accepted: 9 March 2011 /Published online: 10 April 2011

(C) The Author(s) 2011. This article is published with open access at Springerlink.com

\begin{abstract}
Analyses of energy metabolism in human cancer have been difficult because of rapid turnover of the metabolites and difficulties in reducing time for collecting clinical samples under surgical procedures. Utilization of xenograft transplantation of human-derived colon cancer HCT116 cells in spleens of superimmunodeficient NOD/ $\mathrm{SCID} / \mathrm{IL}-2 \mathrm{R} \gamma^{\text {null }}$ (NOG) mice led us to establish an experimental model of hepatic micrometastasis of the solid tumor, whereby analyses of the tissue sections collected by snap-frozen procedures through newly developed microscop-
\end{abstract}

The first two authors equally contributed to this work.

Published in the special issue Biomedical Mass Spectrometry with Guest Editors Hisao Oka and Mitsutoshi Setou.

Electronic supplementary material The online version of this article (doi:10.1007/s00216-011-4895-5) contains supplementary material, which is available to authorized users.

A. Kubo $\cdot$ M. Ohmura $\cdot$ M. Suematsu

Department of Biochemistry, School of Medicine,

Keio University,

35 Shinanomachi, Shinjuku-ku,

Tokyo 160-8582, Japan

M. Ohmura $\cdot$ M. Suematsu $(\bowtie)$

Japan Science and Technology Agency (JST),

Exploratory Research for Advanced Technology

(ERATO) Suematsu Gas Biology Project,

35 Shinanomachi, Shinjuku-ku,

Tokyo 160-8582, Japan

e-mail:msuem@sc.itc.keio.ac.jp

\section{Wakui}

Department of Laboratory Medicine,

School of Medicine,

Keio University,

35 Shinanomachi, Shinjuku-ku,

Tokyo 160-8582, Japan ic imaging mass spectrometry (MIMS) revealed distinct spatial distribution of a variety of metabolites. To perform intergroup comparison of the signal intensities of metabolites among different tissue sections collected from mice in fed states, we combined matrix-assisted laser desorption/ionization time-of-flight imaging mass spectrometry (MALDITOF-IMS) and capillary electrophoresis-mass spectrometry (CE-MS), to determine the apparent contents of individual metabolites in serial tissue sections. The results indicated significant elevation of ATP and energy charge in both

T. Harada $\cdot$ S. Kajihara $\cdot$ K. Ogawa

Technology Research Laboratory, Shimadzu Corporation,

3-9-4 Hikaridai, Seika-cho, Soraku-gun,

Kyoto 619-0237, Japan

H. Suemizu • M. Nakamura

Central Institute for Experimental Animals,

1430 Nogawa, Miyamae-ku, Kawasaki-shi,

Kanagawa 216-0001, Japan

M. Nakamura

Department of Pathology and Regenerative Medicine,

Tokai University School of Medicine,

143 Shimokasuya, Isehara-shi,

Kanagawa 259-1193, Japan

M. Setou

Department of Molecular Anatomy,

Molecular Imaging Frontier Research Center,

Hamamatsu University School of Medicine,

1-20-1 Handayama, Higashi-ku, Hamamatsu-shi,

Shizuoka 431-3192, Japan 
metastases and the parenchyma of the tumor-bearing livers. To note were significant increases in UDP- $N$-acetyl hexosamines, and reduced and oxidized forms of glutathione in the metastatic foci versus the liver parenchyma. These findings thus provided a potentially important method for characterizing the properties of metabolic systems of human-derived cancer and the host tissues in vivo.

Keywords Imaging mass spectrometry (IMS) . Matrix-assisted laser desorption/ionization (MALDI) . Capillary electrophoresis-mass spectrometry (CE-MS) . Tumor-bearing liver $\cdot$ Metabolome $\cdot$ Glycoprotein biosynthesis

\section{Abbreviations \\ CE-MS capillary electrophoresis-mass spectrometry \\ MALDI matrix assisted laser desorption/ionization}

\section{Introduction}

Imaging mass spectrometry (IMS) has enabled us to collect spatial information about a variety of biomolecules, for example phospholipids [1, 2], drugs [3], peptides [4, 5], and metabolites [6-8] in tissue sections in situ. Many kinds of ion sources for imaging mass spectrometry had been developed in recent years. For example, ultraviolet matrixassisted laser desorption/ionization (MALDI) is capable of producing ions from low-molecular-mass compounds, being promising for imaging volatile compounds in fresh ginger samples [9]. Low-molecular-mass metabolites have also been analyzed by other methods including desorption electrospray ionization and desorption/ionization on silicon [10, 11]. In these methods, samples should be dried before image acquisition of metabolomic snapshots to avoid enzymatic degradation of the molecule of interest. On the other hand, laser ablation electrospray ionization or infrared MALDI are other useful methods of molecular imaging that are applicable to water-rich specimens to ionize the analytes $[12,13]$.

In order to acquire reliable images of metabolites in and around cancers in vivo, several lines of methodological consideration are necessary. First, enzymatic reactions in tissue and cell samples should be terminated as soon as possible. Second, soft ionization of the molecules seemed desirable to identify individual peaks of metabolites. Finally, high spatial resolution at cellular levels is required to examine differences in metabolic properties between the cancer foci and the surrounding host tissues. In this context, application of ultraviolet MALDI to freeze-dried tissue samples is suitable for examining cancer metabolism in vivo. Regarding the spatial resolution of the ultraviolet MALDI, the raster scan pitch of the laser application has been restricted by its focusing diameter, in the approximate range 50-200 $\mu \mathrm{m}[7,8]$. Our newly developed UVMALDI-quadrupole ion-trap (QIT)-TOF-MS instrument, that is, a mass microscope with $10-\mu \mathrm{m}$ spatial resolution, overcame this problem of spatial resolution by improvement of the ion source to control the position of the sample stage three-dimensionally with high accuracy $[9,14,15]$. Using 9aminoacridine as a matrix, metabolites, such as high-energy nucleotides and phosphorylated carbohydrates became directly detectable in frozen tissue sections [6]. Since then, various types of tissues including brain and muscles of mice have been analyzed experimentally to dissect molecular mechanisms for metabolic systems in vivo $[7,8,16]$.

The objective of this study was to characterize metabolic systems in and around human-derived solid tumors in vivo. Although distinct properties of cancer metabolism known as Warburg effects have been examined mainly in cultured systems or conventional animal experiments, there are several technical difficulties in characterizing the metabolism of human solid tumors in vivo. First, it is difficult to shorten the time for sampling of tissues to avoid degradation of metabolites with rapid turnover under surgical procedures. Second, because of large variances of the mass intensities of molecules among individual tissue samples, a method to semi-quantify their signals and to compare these among individual samples should be developed. To overcome the first problem, we have herein utilized an experimental model of xenograft transplantation of humanderived colon cancer HCT 116 cell line in superimmunodeficient NOD/SCID/IL-2R $\gamma^{\text {null }}$ (NOG) mice [17]. In this model, we were able to collect snap-frozen samples of the tumor-bearing liver for analyses by using imaging mass spectrometry with high spatial resolution. To solve the second problem, we used serial sections of the liver to determine total amounts of individual metabolites, using CE-MS for data calibration. This method enabled us to carry out intergroup comparison of semi-quantitative metabolite data in and around micrometastases of the human-derived colon cancer. Results obtained in this study revealed that the tumor-bearing liver accelerates its energy metabolism. Furthermore, several metabolites including UDP- $N$-acetyl hexosamine (UDP-HexNAc) and glutathione, and the value of energy charge were significantly elevated in the metastatic foci, suggesting significance of these molecules as marker metabolites enriched in the cancer in vivo.

\section{Experimental}

\section{Chemicals}

9-Aminoacridine (9-AA) was purchased from Merck Schuchardt (Hohenbrunn, Germany). All chemical stan- 
dards were obtained from common commercial sources and dissolved in Milli-Q (Millipore) water, $0.1 \mathrm{~mol} \mathrm{~L}^{-1} \mathrm{HCl}$, or $0.1 \mathrm{~mol} \mathrm{\textrm {L } ^ { - 1 }} \mathrm{NaOH}$ to furnish $10 \mathrm{mmol} \mathrm{L}^{-1}$ or $100 \mathrm{mmol} \mathrm{L}^{-1}$ stock solutions. Distilled water purified by use of a Milli-Q water system was used for all the preparations. Working standard mixtures were prepared by diluting stock solutions with Milli-Q water immediately before injection of samples for CE-MS analysis. The chemicals used were analytical or reagent grades.

Animal model to observe growth of human-derived cancer in vivo

Care and use of laboratory animals were in accordance with the Experimental Animal Committee of Keio University School of Medicine which followed the Guidelines for the Proper Conduct of Animal Experiments by the Science Council of Japan. We herein applied an experimental model using superimmunodeficient NOD/SCID/IL-2R $\gamma^{\text {null }}$ (NOG) mice [18] which lack T-cells, B-cells, and NK cells, enabling xenograft transplantation of the human-derived colon cancer cell line HCT116 transfected with venus, a GFP-mutant gene (HCT116/venus) was injected intrasplenically as described elsewhere $[17,19]$. To our knowledge, the system using NOG mice serves as a reliable experimental model in which human-derived cancer cell lines transplanted in vivo generate metastases forming solid tumors in organs including the liver in vivo with greater reproducibility than the conventional model using NOD mice which lack T-cells and B-cells but not NK cells [17]. In the intrasplenic implantation model using HCT116/venus, micrometastases of the cancer occurred mostly in periportal regions of the liver within one week, extending their size over the following three weeks [19]. In this study, male mice at 11-13 weeks were fed with Laboratory chow and allowed free access to water. They were anesthetized by use of pentobarbital sodium, and their abdominal walls were cut to exteriorize the portion of the liver lobules for snap-frozen procedures using liquid nitrogen as described elsewhere [20, 21]. The livers of the xenografttransplanted NOG mice used for the analyses were collected under fed conditions two weeks after the xenograft transplantation, unless otherwise mentioned.

\section{Tissue preparation}

A schematic diagram of a standard experimental design typical of tissue section application can be seen in the Electronic Supplementary Material (Fig. S1). The liver tissues snap-frozen in liquid nitrogen were dissected to prepare cryosections with $5-\mu \mathrm{m}$ thickness by use of a cryostat (CM 1900; Leica Microsystems, Wetzlar, Germany). The sections were thaw-mounted on indium-tin oxide (ITO) slides (8-12 ohm/sq; Sigma, Chiba, Japan) and were dried in silica gel-containing plastic tubes then sprayed with 9-aminoacridine (9-AA, $20 \mathrm{mg}$ in $4 \mathrm{~mL} \mathrm{70 \%} \mathrm{MeOH)} \mathrm{by}$ use of a 0.2-mm nozzle caliber airbrush (Procon boy FWA Platinum; Mr Hobby, Tokyo, Japan) to conduct MALDI-IMS in negative ion mode. Adjacent sections were fixed with $10 \%$ buffered formalin (Nacalai Tesque, Kyoto, Japan) and stained with the Masson trichrome staining reagent kit according to the manufacturer's procedures (Muto Pure Chemicals, Tokyo, Japan). The other frozen tissues were used for metabolome analyses using CE-MS.

Instruments

An atmospheric pressure MALDI-QIT-TOF-MS equipped with a 355-nm Nd:YAG laser and a built-in fluorescence microscope furnished mass images with high spatial resolution of $10 \mu \mathrm{m}$, as described for a prototype IMS machine [9] (Mass Microscope; Shimadzu, Kyoto, Japan). The microscope was also equipped with a 120-W metal halide light source (X-Cite 120PC-Q; Lumen Dynamics Group, Ontario, Canada) and a fluorescence mirror unit (UMGFPHQ; Olympus, Tokyo, Japan) that enabled us to visualize venus-associated green fluorescence from metastatic foci in tissue slices before matrix application. This system thus enabled us to collect both fluorescence images and mass images from the same tissue slices. Before applying matrices, a fluorescence image of the analyte was acquired and followed by recording the location of the specimen on the $X Y Z$ stage. ITO slides with the sample holder were removed from the $X Y Z$ stage, and sprayed uniformly with $4 \mathrm{~mL}$ 9-AA solution. After application of the matrix, the sample holder was placed back on the $X Y Z$ stage, which was adjusted to exactly the same location. The mass spectra of the designated areas on a specimen photographed before matrix application were acquired in the negative-ion mode. The data collected through the microscopic system were digitally processed according to the method described later, in the section "Determination of the apparent contents of metabolites based on data calibration by CE-MS". Experiments using capillary electrophoresis electrospray ionization mass spectrometry (CE-MS) were performed with a capillary electrophoresis system equipped with an air pressure pump, MSD quadrupole MS, isocratic HPLC pump, and CE-MS adapter kit (Agilent Technologies, Waldbronn, Germany) [20, 22]. All system control, data acquisition, and MSD data evaluation were performed using Agilent G2201A ChemStation software for CE-MSD.

Conditions for MALDI imaging and tandem MS analyses

All the MALDI imaging experiments and tandem MS experiments were carried out in negative-ion mode using a prototype mass microscope. The laser power was adjusted 
to the desired intensities. MALDI mass spectra were acquired under the conditions laser frequency $800 \mathrm{~Hz}$ and scanning mass range from $\mathrm{m} / \mathrm{z} 260$ to $\mathrm{m} / \mathrm{z} 900$. Regions of the tissue samples exposed to the laser irradiation were determined by light and fluorescence microscopic observations. A raster scan on the tissue surface was performed automatically $(250 \times 250$ spots per scan). Laser irradiation was repeated 160 times for the individual spots. The spatial interval of data points was $10 \mu \mathrm{m}$, giving 62,500 data points in total for each section. Tandem MS analyses were performed to determine the structures of metabolites at appropriate laser energy, argon gas percentage, and collision-induced dissociation (CID) energy conditions. To identify metabolites detected in MALDI-IMS, we searched the Human Metabolome Database (http://hmdb.ca) and performed tandem MS analyses, comparing the tandem mass spectra with those from standard reagents using 9-AA as the matrix of choice in negative mode. The distribution of biomolecules that were identifies by $\mathrm{MS}^{2}$ analysis were visualized as mass images using BioMap software (http:// www.maldi-msi.org) from Novartis (Basel, Switzerland).

Determination of the apparent contents of metabolites based on data calibration by CE-MS

To compare differences in contents of many different metabolites in multiple tissue slices collected from different subjects, we conducted CE-MS-based metabolome analyses of tissue lysates collected from the same snap-frozen liver samples for IMS. As reported previously [20-22], CE-MS enabled us to quantitatively determine more than 2,000 different metabolites at once in small amounts of a single liver sample. Small amounts of the frozen tissue were collected from thick section that was adjacent to the section for IMS. The CE-MS samples weighing approximately $300 \mathrm{mg}$ wet weight was ground using a multi-bead shocker (model MB755U; Yasui Kikai, Osaka, Japan) with frozen metal granules, and $1 \mathrm{~mL}$ methanol containing internal standards (300 $\mu \mathrm{mol} \mathrm{L}^{-1}$ each of L-methionine sulfone and 2morpholinoethanesulfonic acid (MES)) was added. After addition of $0.5 \mathrm{~mL}$ deionized water and $1 \mathrm{~mL}$ chloroform, the mixture was centrifuged at $15,000 \mathrm{rpm}$ for $15 \mathrm{~min}$ at $4{ }^{\circ} \mathrm{C}$. The upper aqueous layer of the sample was filtered through the 5-kDa cutoff filter (Millipore, Tokyo, Japan) by centrifugation to remove protein components. The filtrate was lyophilized and dissolved in $200 \mu \mathrm{L}$ deionized water containing reference compounds $\left(200 \mu \mathrm{mol} \mathrm{L}^{-1}\right.$ each of 3-aminopyrrolidine and trimesate). The amounts of metabolites were normalized by liver weight of the original tissue samples. Assuming that $1 \mathrm{mg}$ wet tissue was equivalent to $1 \mu \mathrm{L}$, apparent contents of individual metabolites were calculated for the data calibration in imaging mass microscopy by computer-assisted data processing in the later session.
To semi-quantify apparent contents of individual metabolites mapped on the images, regions of interest (ROIs) for the cancer xenografts and the liver parenchyma were selected by careful visual inspection of fluorescence and light microscopic images. Averaged spectra from each ROI were generated and statistically analyzed by use of the laboratory-written software SIMtools (Shimadzu Imaging Mass spectrometry toolbox for MATLAB). The MannWhitney U-test was used to compare the ion signal intensity between the tumor and the liver parenchyma.

To calculate apparent contents of metabolites in IMS, mass signals covering $\mathrm{m} / \mathrm{z}$ between 260 and 620 were collected from $250 \times 250$ spots on a tissue sample. Direct comparison of metabolite ion signals among different slices requires realistic transformation of acquired mass signals of a metabolite in absolute terms, for example tissue contents in nmol g ${ }^{-1}$ tissue. To that effect, apparent contents of a specific metabolite in the ROI designated as liver parenchyma ([ $\left.\left.C_{\text {Liver }}\right]_{\text {app }}\right)$ or tumor ([ $\left.\left.C_{\text {Tumor }}\right]_{\text {app }}\right)$ were calculated by use of Eqs. 1, 2, and 3:

$\left[C_{\text {Tumor }}\right]_{\text {app }}=\left[C_{\text {Average }}\right] \times\left(I_{\text {Tumor }} / I_{\text {Average }}\right)$

$\left[C_{\text {Liver }}\right]_{\text {app }}=\left[C_{\text {Average }}\right] \times\left(I_{\text {Liver }} / I_{\text {Average }}\right)$

$$
\begin{aligned}
I_{\text {Average }}= & \left(I_{\text {Liver }} \times O_{\text {Liver }}\right) / 100 \\
& +\left(I_{\text {Tumor }} \times O_{\text {Tumor }}\right) / 100
\end{aligned}
$$

where $\left[C_{\text {Average }}\right]$ denotes the amount of a metabolite of interest in the whole tissue section for IMS ( $\mathrm{nmol} \mathrm{g}^{-1}$ tissue). $I_{\text {Tumor }}, I_{\text {Liver, }}$, and $I_{\text {Average }}$ are MALDI signal intensities measured in regions of tumor metastases, liver parenchyma, and the whole tissue section, respectively. $O_{\text {Tumor }}$ and $O_{\text {Liver }}$ are the percentage areas of tumor regions and liver parenchyma, respectively, in tumor-bearing livers. These indices were designated by the green fluorescence intensities acquired by fluorescence microscopy of the whole frozen section using Image J software (NIH, http://rsbweb.nih.gov/ $\mathrm{ij} /$ index.html). Assuming that percentages of tumor-bearing regions in the tissue sections are comparable with those of the metastases in the adjacent tissue samples for the CE-MS measurements, the values of $\left[C_{\text {Average }}\right]$ can be replaced by $\left[C_{\mathrm{CE}-\mathrm{MS}}\right]$, the amounts of the metabolites of interest in the tissue samples for CE-MS, as seen in Eqs. 1' and 2'.

$\left[C_{\text {Tumor }}\right]_{\text {app }}=\left[C_{\mathrm{CE}-\mathrm{MS}}\right] \times\left(I_{\text {Tumor }} / I_{\text {Average }}\right)$

$\left[C_{\text {Liver }}\right]_{\mathrm{app}}=\left[C_{\mathrm{CE}-\mathrm{MS}}\right] \times\left(I_{\text {Liver }} / I_{\text {Average }}\right)$

In order to calculate the values for $\left[C_{\text {Tumor }}\right]_{a p p}$ and $\left[C_{\text {Liver }}\right]_{\text {app }}, I_{\text {Tumor }}, I_{\text {Liver }}$, and $I_{\text {Average }}$ were determined in 
three different ROIs in a single tissue specimen, while the corresponding value of $\left[C_{\mathrm{CE}-\mathrm{MS}}\right]$ was collected from a portion of the tissue adjacent to the samples for IMS. A set of mass-imaging data paired with CE-MS data in a single animal were repeated for four separate animals.

To construct an ion-content map, data collected by MALDI-QIT-MS were saved and digitally processed to be converted to Analyze format, a common multidimensional biomedical imaging format file. The Analyze format file was processed to construct an ion-content map, where the relationship between the apparent content of a metabolite of interest at a pixel " $i$ " $(C i)$ and the MALDI signal intensity in the same pixel $\left(I_{(i)}\right)$ was expressed by Eq. 4:

$C i=\left[C_{\mathrm{CE}-\mathrm{MS}}\right] \times\left(I_{(i)} / I_{\text {Average }}\right)$

Validation of the calibrated data in IMS by analysis of high-energy adenylates in vivo

Reliability of the calibrated data seemed to depend on multiple factors including the quality of CE-MS-based metabolome analysis and that of tissue-sampling procedures in the snap-frozen processes. In accordance with our previous studies [7], we determined apparent contents of ATP, ADP, and AMP by IMS combined with CE-MS. Based on these data, apparent energy charge of individual pixels were calculated by use of Eq. 5:

Apparent energy charge $\left(E C_{\text {app }}\right)=$

$$
\left([\mathrm{ATP}]_{\mathrm{app}}+1 / 2[\mathrm{ADP}]_{\mathrm{app}}\right) /\left([\mathrm{ATP}]_{\mathrm{app}}+[\mathrm{ADP}]_{\mathrm{app}}+[\mathrm{AMP}]_{\text {app }}\right)
$$

Statistics

Differences between mean values for two groups were statistically analyzed by use of the Mann-Whitney $U$-test. $P<0.05$ was considered statistically significant.

\section{Results and discussion}

Detection of metabolites from normal mouse liver tissue

As shown in Fig. 1, MALDI-MS analyses in the negativeion mode using 9-AA as the matrix revealed the presence of many signals including nucleotides on the liver tissue frozen section with minimal interference from the matrix, as reported by Edwards and Kennedy [6]. Among these peaks, 16 intense mass peaks were assigned using their exact masses to nucleotides, nucleotide-sugar conjugates, 2,3bisphosphoglycerate (2,3-BPG), taurocholate, and both reduced and oxidized forms of glutathione. Assignment of these metabolites was verified by structural analysis of individual peaks using tandem MS. In the region adjacent to those for mass-imaging experiments in the same section, the peaks generated on the tissue surface were identified by fragmentation using MALDI-QIT-TOF MS [9]. Table 1 summarizes the results of MS and $\mathrm{MS}^{2}$ analyses. Among the individual mass peaks of nucleoside-monophosphate, the ribose-phosphate structure was present (211.0 fragment ions and neutral losses of adenine, uridine, and guanidine from precursor ions respectively). Among the peaks of nucleotide-sugar conjugates, fragment ions derived from the UDP structure were confirmed (403.0 fragment ions and
Fig. 1 Typical MALDI mass spectrum of the mouse liver collected from the "mass microscope" a MALDI-IT-TOF analyzer in the negative-ion mode. The signals ranging from $m / z=260$ to 620 were collected. Mass spectra of metabolites were obtained by integrating $10,000,000$ consecutive laser shots (160 shots per subspectrum and 62,500 subspectra). Peaks identified in this study are labeled; that originating from the 9-AA matrix is indicated by an asterisk

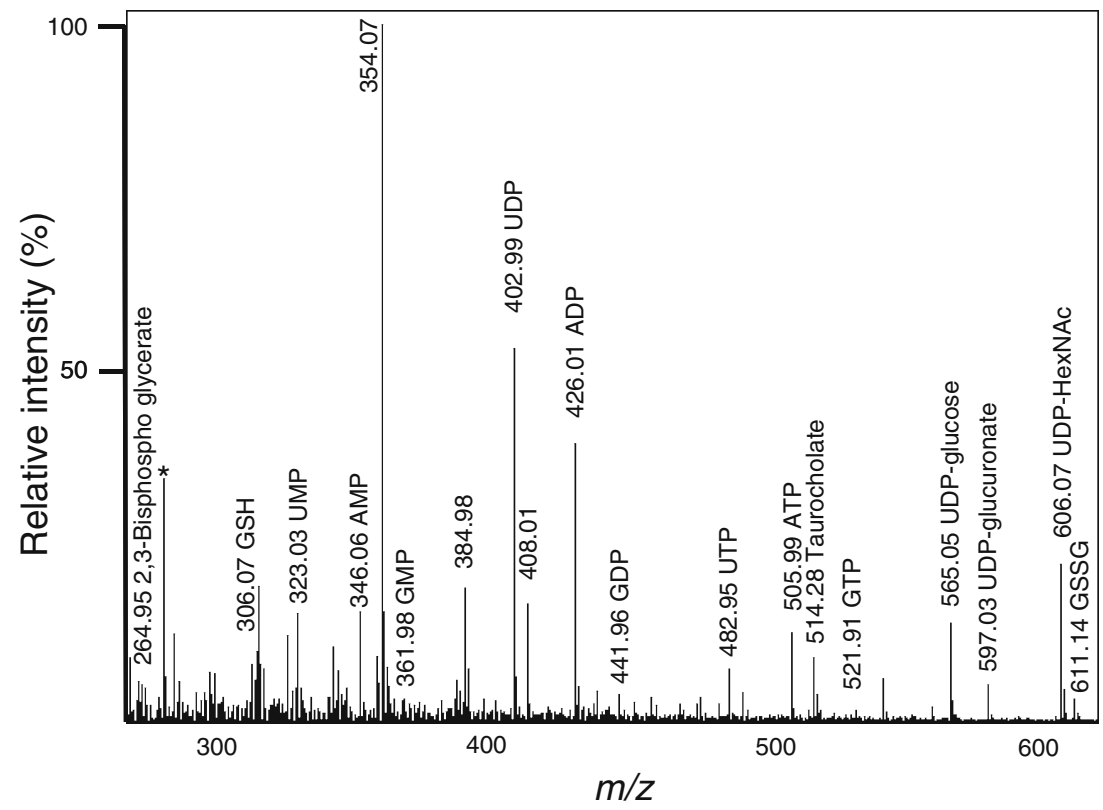


Table 1 List of metabolites identified in the study, with their respective deprotonated $\mathrm{m} / \mathrm{z}$ values and $\mathrm{MS}^{2}$ ion species in the negative mode

\begin{tabular}{lllll}
\hline No. & $\begin{array}{l}{[\mathrm{M}-\mathrm{H}]^{-}} \\
(\mathrm{m} / \mathrm{z})\end{array}$ & Fragments observed in $\mathrm{MS}^{2}$ & Formula & Identified \\
\hline 1 & 264.95 & $246.973,179.043,166.955$ & & \\
2 & 306.07 & $254.087,179.050,143.048,158.932,210.092,288.858,120.041$ & $\mathrm{C}_{3} \mathrm{H}_{8} \mathrm{O}_{10} \mathrm{P}_{2}$ & 2,3-Bisphosphoglycerate \\
3 & 323.02 & $211.006,280.028,138.983,158.922$ & $\mathrm{C}_{10} \mathrm{H}_{17} \mathrm{~N}_{3} \mathrm{O}_{6} \mathrm{~S}$ & Glutathione (GSH) \\
4 & 403.00 & $304.984,158.907,384.935,322.989,290.931,272.920$ & $\mathrm{C}_{9} \mathrm{H}_{13} \mathrm{~N}_{2} \mathrm{O}_{9} \mathrm{P}$ & UMP \\
5 & 482.93 & $384.927,272.919,176.913,158.905$ & $\mathrm{C}_{9} \mathrm{H}_{14} \mathrm{~N}_{2} \mathrm{O}_{12} \mathrm{P}_{2}$ & UDP \\
6 & 346.05 & $211.000,150.985,134.049,194.051$ & $\mathrm{C}_{9} \mathrm{H}_{15} \mathrm{~N}_{2} \mathrm{O}_{15} \mathrm{P}_{3}$ & UTP \\
7 & 426.02 & $328.044,134.053,407.999,329.037,272.950,290.945$ & $\mathrm{C}_{10} \mathrm{H}_{14} \mathrm{~N}_{5} \mathrm{O}_{7} \mathrm{P}$ & AMP \\
8 & 505.98 & $408.022,272.958,158.922,176.933,392.003$ & $\mathrm{C}_{10} \mathrm{H}_{15} \mathrm{~N}_{5} \mathrm{O}_{10} \mathrm{P}_{2}$ & ADP \\
9 & 361.98 & $210.985,260.838,158.908$ & $\mathrm{C}_{10} \mathrm{H}_{16} \mathrm{~N}_{5} \mathrm{O}_{13} \mathrm{P}_{3}$ & ATP \\
10 & 441.96 & $343.992,150.025,158.909$ & $\mathrm{C}_{10} \mathrm{H}_{14} \mathrm{~N}_{5} \mathrm{O}_{8} \mathrm{P}$ & GMP \\
11 & 521.91 & $423.940,272.924,176.911,158.906,229.979$ & $\mathrm{C}_{10} \mathrm{H}_{15} \mathrm{~N}_{5} \mathrm{O}_{11} \mathrm{P}_{2}$ & GDP \\
12 & 565.03 & $323.024,306.072,402.984,211.000,384.977$ & $\mathrm{C}_{10} \mathrm{H}_{16} \mathrm{~N}_{5} \mathrm{O}_{14} \mathrm{P}_{3}$ & GTP \\
13 & 579.02 & $323.027,402.997,254.996,305.011,210.994$ & $\mathrm{C}_{15} \mathrm{H}_{24} \mathrm{~N}_{2} \mathrm{O}_{17} \mathrm{P}_{2}$ & UDP-glucose (UDP-Hexose) \\
14 & 606.06 & $384.9445,402.996,282.045,272.956,323.038,305.017,362.008$ & $\mathrm{C}_{15} \mathrm{H}_{22} \mathrm{~N}_{2} \mathrm{O}_{18} \mathrm{P}_{2}$ & UDP-glucuronic acid \\
15 & 611.14 & $306.071,272.086,338.058,254.079,482.103,304.058,288.058,593.121$ & $\mathrm{C}_{17} \mathrm{H}_{27} \mathrm{~N}_{3} \mathrm{O}_{17} \mathrm{P}_{2}$ & UDP-GlcNAc (UDP-HexNAc) \\
\hline
\end{tabular}

neutral loss of each hexose, glucuronic acid, and $N$ acetylhexosamines). All the assigned and verified metabolites in Table 1 were compared with commercial standard compounds under appropriate MALDI and CID tandem MS conditions.

Intralobular distributions of metabolites in the liver

Figure 2 shows the tissue distribution of metabolites in the control fed liver of NOG mice. As seen in Figs. 2a and b, by use of Masson trichrome staining we were able to distinguish portal and central venules by judging the presence of bile ducts as a typical structure of the portal triad. Worthy of note is that the intraluminal space of the portal $(\mathrm{P})$ and central venules indicated strong signals of 2,3-BPG (Fig. 2d) whereas taurocholate, the taurineconjugated form of cholic acid, was evident in bile ducts (B) adjacent to the portal venules and periportal parenchyma, showing a typical P-P bridging pattern (Fig. 2e). On the other hand, UDP-glucuronate, a substrate for glucuronidization of xenobiotic compounds was localized mainly in pericentral regions, with a pattern distinct from that of taurocholate (Fig. 2f). Such an intralobular microanatomical relationship among periportal and pericentral regions and the microvessels was well demonstrated by superimposition of taurocholate ( $\mathrm{m} / \mathrm{z}$ 514.28; green in Figs. $2 \mathrm{~g}$ and $\mathrm{h}$ ) with 2,3-BPG ( $m / z$ 264.95; red in Fig. $2 \mathrm{~g})$ or UDP-glucuronate ( $\mathrm{m} / \mathrm{z}$ 579.03; red in Fig. 2h), respectively. In contrast, an intralobular pattern of UDP-HexNAc was distinct, distributing homogeneously over the whole liver parenchyma (Fig. 2i). These results suggest that several different metabolites such as 2,3-BPG, taurocholate, and UDPglucuronate visualized by use of this method serve as reliable markers to distinguish vascular structures and periportal and pericentral regions in hepatic zonation. Such results were also consistent with previous observations as follows. First, 2,3-BPG occurs abundantly in red blood cells, being at approximately $5 \mathrm{mmol} \mathrm{L}^{-1}$ [23]. Secondly, taurocholate is synthesized and excreted to bile canaliculi around periportal hepatocytes [24, 25], whereas UDPglucuronate is biosynthesized predominantly in pericentral hepatocytes, which had a large capacity for xenobiotic detoxification [26, 27]. Our approach to analysis of metabolites at the microscopic level, serves as a powerful tool to investigate intralobular heterogeneity in metabolic systems in the liver.

Distinct properties of energy metabolism between cancer metastases and liver parenchyma

Figure 3 shows representative pictures of high-energy adenylate phosphates in liver bearing colon cancer metastases under fed conditions. As seen in Figs. 3a and b, the micrometastases were clearly identified by green fluorescence generated by venus-expressing HCT116 cells. Figure $3 c$ depicts mapping of apparent energy charge that was calculated from images of AMP, ADP, and ATP (Figs. 3d, e, and f, respectively). Worthy of note was that intralobular distribution of these three adenylates appeared almost homogeneous, whereas the energy charge composed from these images had a heterogeneous pattern, being greater at the sites of cancer metastasis than in the liver 
Fig. 2 In situ mass spectrometric imaging of metabolites in the control liver of an NOG mouse. a An optical image of a section stained with Masson's trichrome solutions. The stained section was adjacent to that was used for IMS. Scale bar: $500 \mu \mathrm{m}$, $B$ :bile duct, $P$ :portal venule. b the image with greater magnification of the area indicated by the white square in a. Collagen fibers in the portal triad were stained in blue. $B$ :bile duct, $P$ :portal venule. c Lightmicroscopic image of frozen liver section used in mass spectrometric imaging that was captured before matrix application. Scale bar: $500 \mu \mathrm{m}$. d An ion image of 2,3-bisphosphoglycerate $(2,3-\mathrm{BPG})$ at $\mathrm{m} / \mathrm{z}=$ 264.95 and e taurocholate at $\mathrm{m} / \mathrm{z}=514.28$, f UDP-glucuronate at $m / z=579.03$. g The overlaid image of 2,3-BPG (red) and taurocholate (green). h UDPglucuronate (red) and taurocholate (green). i UDP-HexNAc at $m / z=606.06$. A representative set of pictures from four separate experiments

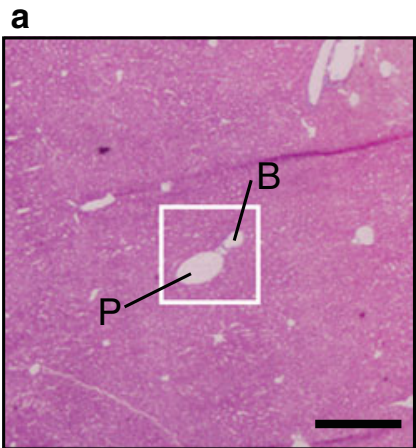

Masson trichrome stain

d

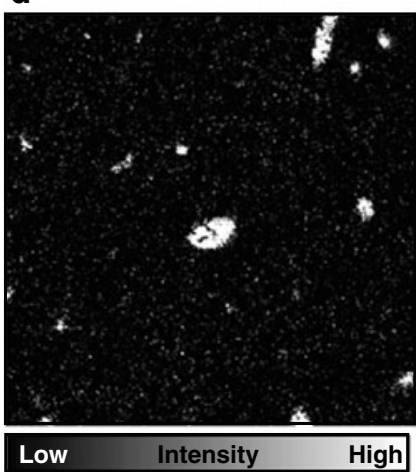

$m / z 264.95$

2,3-Bisphosphoglycerate

g

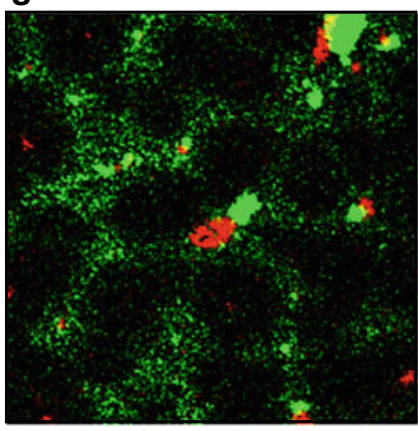

Green: $m / z 514.28$

Red: $m / z 264.95$ b

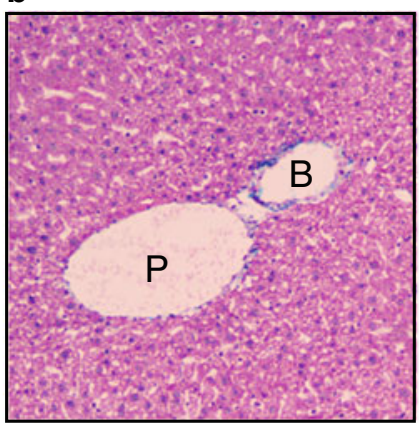

Masson trichrome stain

e

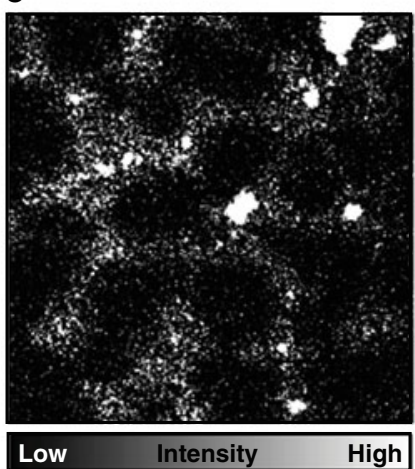

$m / z 514.28$

Taurocholate

h

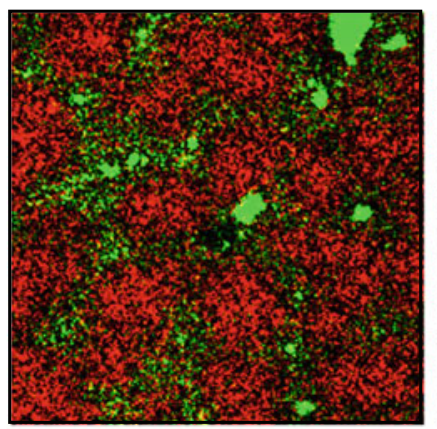

Green: $m / z 514.28$

Red: $m / z 579.03$
C

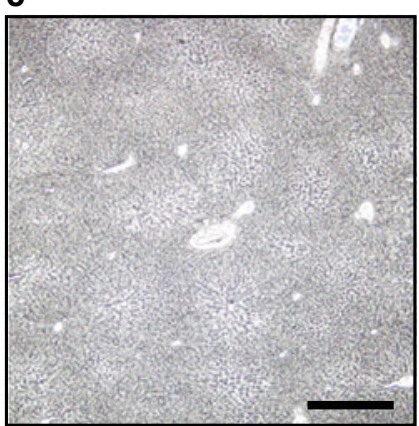

Light microscope

f

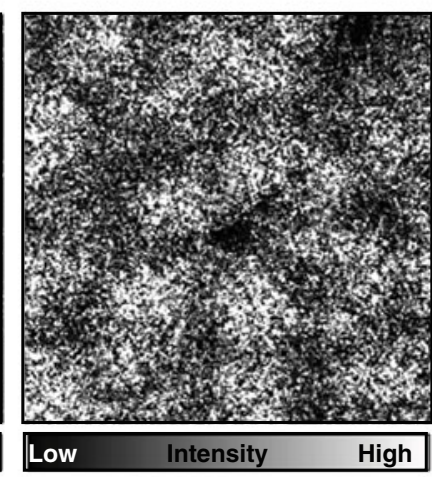

$\mathrm{m} / \mathrm{z} 579.03$

UDP-glucuronate

i

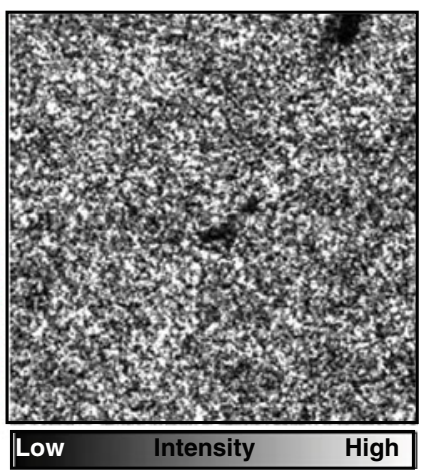

$\mathrm{m} / \mathrm{z} 606.06$

UDP-N-acetylhexosamine parenchyma. So far as judged from the intensities, the apparent energy charge in the metastatic foci was approximately 0.8 , while that in the parenchyma was $0.5-0.7$, suggesting that the foci accelerate generation of highenergy adenylate phosphates. Of importance were semiquantitative values of $[\mathrm{ATP}]_{\mathrm{app}}$ in the tumor-bearing liver (Fig. 3f), in the range of approximately $1-2 \mu \mathrm{mol} \mathrm{g}^{-1}$ liver, levels reasonably comparable with previous observations that determined absolute ATP contents under the snapfrozen procedures $[20,21]$. Because intracellular levels of ATP are in the $\mathrm{mmol} \mathrm{L}^{-1}$ range, current procedures for data calibration seemed to reproduce reliable intracellular concentrations of metabolites with a rapid turnover.
Glutathione and UDP-HexNAc serve as marker metabolites enriched in the cancer xenografts

Figure 4 shows representative pictures of UDP-HexNAc, reduced (GSH), and oxidized (GSSG) forms of glutathione. As seen in Figs. 4a-c, UDP-HexNAc was predominantly high in the tumor metastases. GSH and GSSG signals were also greater in the metastatic foci, but with a different pattern from that of UDP-HexNAc (Figs. 4d-f). When focusing on individual foci, UDP-HexNAc and GSH seemed to be demarcated from each other, suggesting these molecules occur in a spatially heterogeneous manner in an individual metastatic focus. 
Fig. 3 Representative pictures indicating high-energy adenylate phosphates and apparent energy charge in the tumorbearing livers of NOG mice. a A light-microscopic photograph of intrasplenically injected HCT116 colon cancer cell b A green fluorescence image of the same region shown in (a). Scale bar: $500 \mu \mathrm{m}$. c A heat map of apparent energy charge calculated from apparent contents of tumor bearing-liver. d-f Heat maps of [AMP $]_{\mathrm{app}}$, $[\mathrm{ADP}]_{\mathrm{app}}$, and $[\mathrm{ATP}]_{\mathrm{app}}$, respectively. A representative set of pictures from four separate experiments xenografts. Scale bar: $500 \mu \mathrm{m}$.

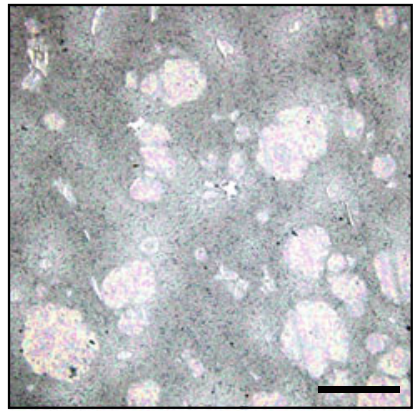

Light microscopic image

d

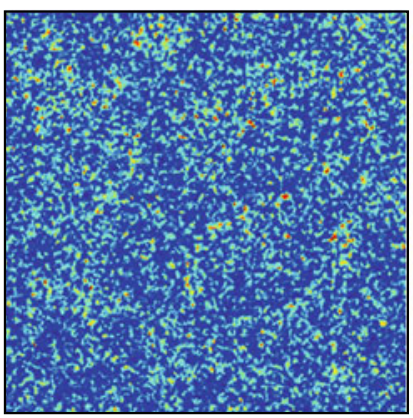

0 [AMP] ${ }_{\text {app }} \mu \mathrm{mol} / \mathrm{g}$ tissue

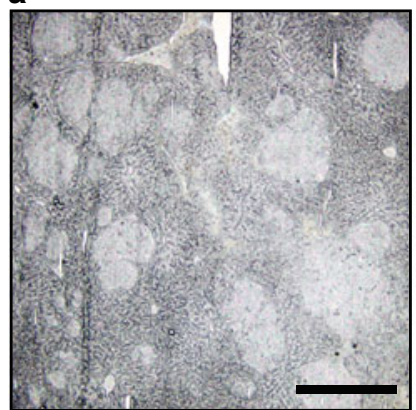

Light microscopic image

Fig. 4 Glutathione and UDPHexNAc as marker metabolites enriched in hepatic cancer metastasis. a Light-microscopic photograph of intrasplenically injected HCT116 colon cancer cell xenografts in the liver of NOG mice. Scale bar: $500 \mu \mathrm{m}$. b A green fluorescence image of the same specimen shown in (a). Scale bar: $500 \mu \mathrm{m}$. c An apparent contents map of UDP-HexNAc at $\mathrm{m} / \mathrm{z}$ 606.06. d, e Apparent contents maps of the reduced type of glutathione (GSH) at $\mathrm{m} / \mathrm{z} 306.07$, and oxidized glutathione (GSSG) at $\mathrm{m} / \mathrm{z}$ 611.14. f $[\mathrm{GSH}]_{\mathrm{app}} /[\mathrm{GSSG}]_{\mathrm{app}}$ ratio imaging in the same microscopic field plotted as a heat map. A representative set of pictures from four separate experiments

d a

b

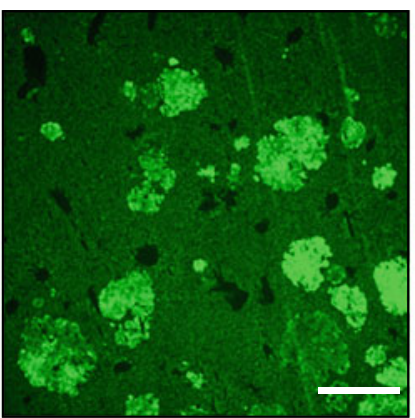

Fluorescent microscopic image venus (green): Tumor

e

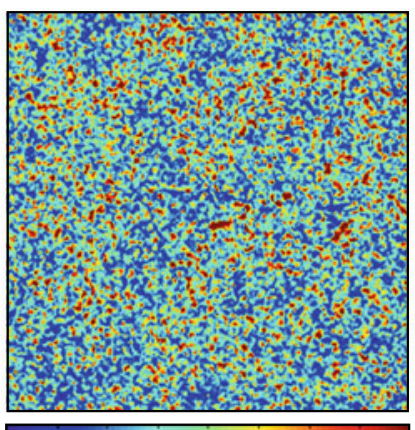

0

[ADP] $]_{\text {app }} \mu \mathrm{mol} / \mathrm{g}$ tissue

b

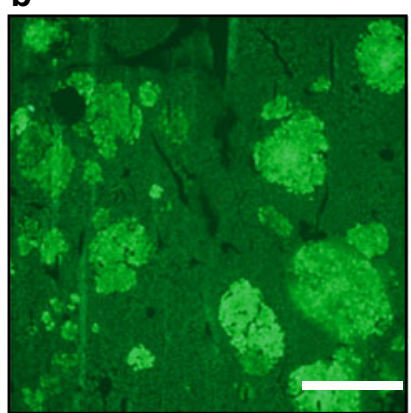

Fluorescent microscopic image venus (green): Tumor

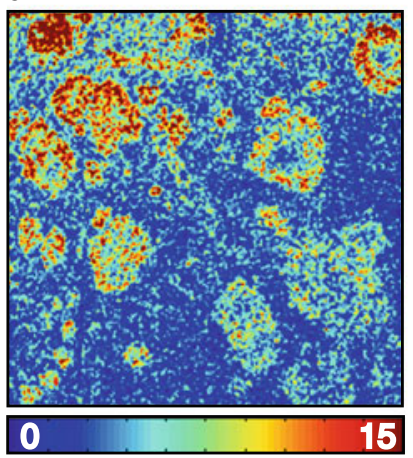

[GSH] app $\mu \mathrm{mol} / \mathrm{g}$ tissue e

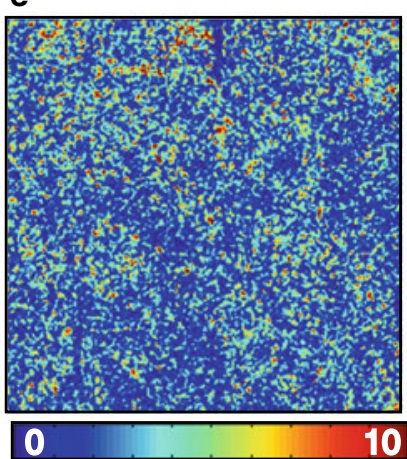

[GSSG]app $\mu \mathrm{mol} / \mathrm{g}$ tissue
C

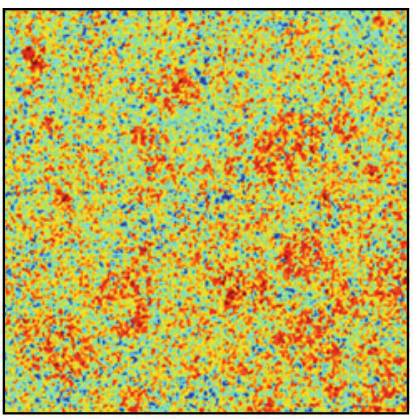

0 0.8

Energy charge

f

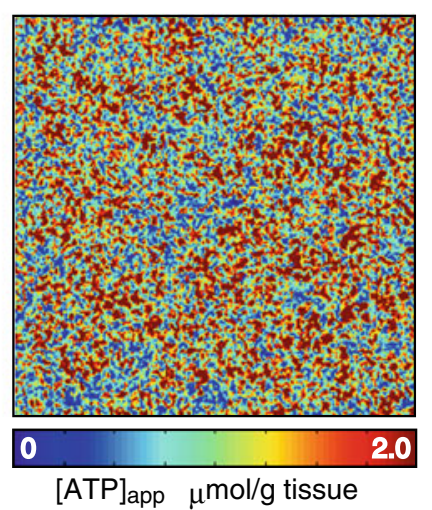

C

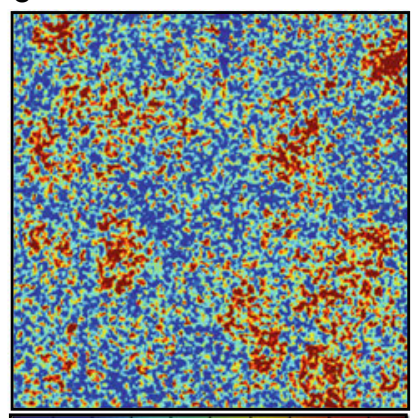

1.0

[UDP-HexNAc]app $\mu \mathrm{mol} / \mathrm{g}$ tissue

f

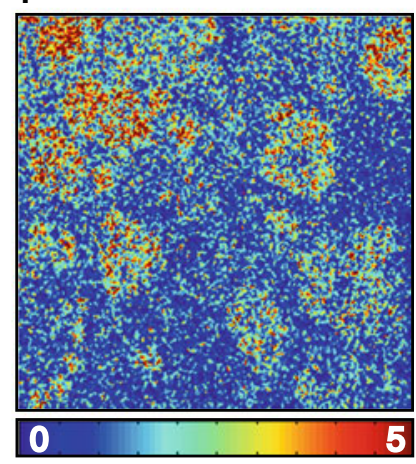

$[\mathrm{GSH}]_{\mathrm{app}} /[\mathrm{GSSG}]_{\mathrm{app}}$ 
Figure 5 illustrates semiquantitative comparison of these metabolites among different groups. As seen in Fig. 5a showing data collected from CE-MS, amounts of UDPHexNAc in the tumor-bearing liver (tumor plus liver) were significantly greater than those in the control liver. On the other hand, amounts of GSH and GSSG were not significantly different between the groups. When MALDI-MS intensity ratios in IMS for these metabolites were analyzed (Fig. 5b), UDP-HexNAc, GSH, and GSSG were significantly elevated in the metastatic foci compared with those measured in the hepatic parenchyma. Such a trend was reproducible in the calibrated data showing apparent contents of these metabolites (Fig. 5c), suggesting that both forms of glutathione occurred prominently in the metastatic foci to greater extents than in the host liver parenchyma.

In the control liver, UDP-HexNAc which is an important substrate for polysaccharide chains including hyaluronic acid, was homogeneously distributed over the liver (Fig. 2i). As seen in Fig. 4, on the other hand, the colon cancer xenografts exhibited greater accumulation than the liver parenchyma. Ishimoto et al. and our laboratory have recently provided evidence that $\mathrm{CD} 44$, the hyaluronic acid receptor expressed on cancer stem cells, is responsible for stabilization of the cystine transporter $\mathrm{xCT}$ that accelerates uptake of cysteine and glutathione to increase their antioxidative capacity against cell death [28]. Because GSH detoxifies electrophiles through their conjugation by glutathione $S$-transferases, such accumulation of the thiol might contribute to survival of the cancer cells against anti-cancer reagents $[29,30]$. Up to now, it remains still difficult to visualize amino acids globally by MALDI-MS with high spatial resolution. Improvement of the spatial resolution in the current IMS system together with development of the method to visualize amino acid metabolism deserves further studies provided that resources and mechanisms for delivery of amino acids to maintain GSH in cancer cells are revealed.

\section{Conclusion}

Comparison of differences in metabolomic profiles between control livers and those bearing metastatic foci of the human colon cancer enabled us to reveal distinct metabolic features between the two regions. IMS using 9aminoacridine enabled us to detect nucleotides, lipids, and several aminosugars. The metastatic human colon cancer xenografts displayed remarkable accumulation of UDPHexNAc and glutathione in vivo, serving as marker metabolites. Newly developed IMS combined with CEMS measurements serves as a potentially reliable method to perform intergroup comparison of varied metabolites among different tissue specimens. Future investigation is a

CE-MS

UDP-HexNAc
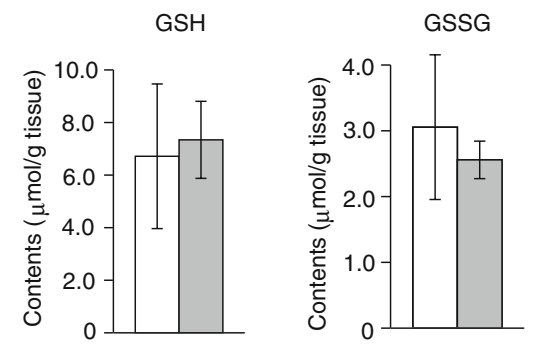

b

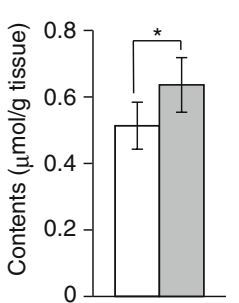

MALDI-MS intensity ratio
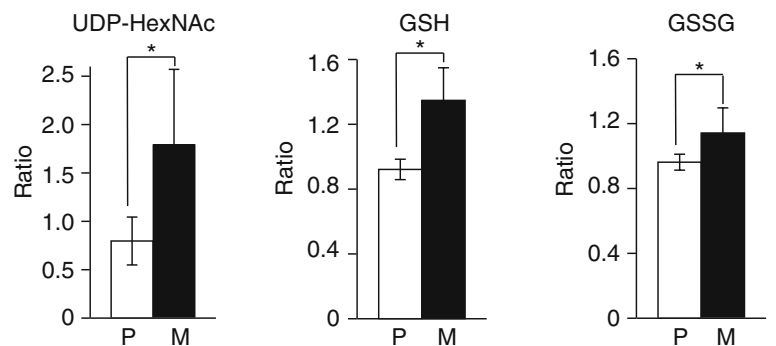

C Apparent contents in liver parenchyma (P) and metastases (M).
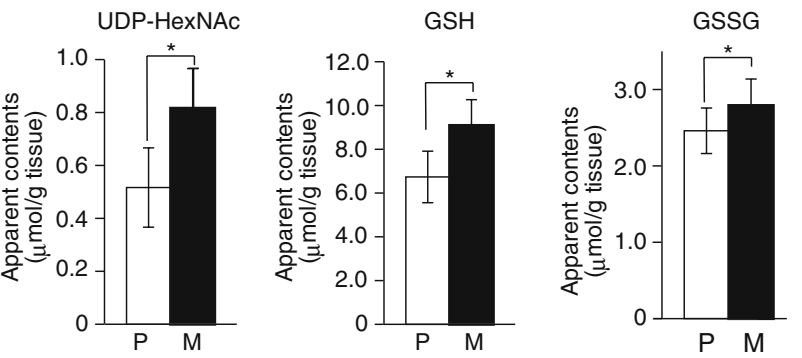

Fig. 5 Differences in contents of UDP-HexNAc, and reduced $(G S H)$ and oxidized (GSSG) glutathione in the tumor-bearing-NOG liver. a Differences in CE-MS data between the control (open columns) and the tumor-bearing livers (dark columns) of NOG mice. b The MALDI-MS signal intensity ratio of UDP-HexNAc, GSH, and GSSG in the parenchyma ( $P$; open columns) and metastatic foci $(M$; closed columns) versus the average MALDI-MS intensity of the ROIs as a whole. Bars indicate SD. ${ }^{*} P<0.05$ according to Mann-Whitney $U$-tests. c Apparent amounts of UDP-HexNAc, GSH, and GSSG in the parenchyma $(P$; open columns) and metastatic foci $(M$; closed columns). Bars indicate SD from four separate experiments. ${ }^{*} P<0.05$, Mann-Whitney $U$-tests

necessary to establish methodology for use of the current technique in diagnostic medicine.

Acknowledgements The authors are grateful to Masaaki Matsuura (Japan Federation of Cancer Research) and all the member of the SENTAN project (Shimadzu Corporation), Kenji Kawai (Central Institute for Experimental Animals), and members of Suematsu Laboratory for providing considerable support for our research. The authors acknowledge support for this work by a Grant-in-Aid for SENTAN from the Japan Science and Technology Agency (to M.Se. and M.W) and from the JST ERATO Suematsu Gas Biology Project (to M.Su). Design of CE-MS-based metabolome analysis in this study was supported by Research and Development of the Next-Generation Integrated Simulation of Living Matter, a part of the Development and Use of the Next-Generation Supercomputer Project of MEXT. 
Open Access This article is distributed under the terms of the Creative Commons Attribution Noncommercial License which permits any noncommercial use, distribution, and reproduction in any medium, provided the original author(s) and source are credited.

\section{References}

1. Sugiura Y, Konishi Y, Zaima N, Kajihara S, Nakanishi H, Taguchi R, Setou M (2009) Visualization of the cell-selective distribution of PUFA-containing phosphatidylcholines in mouse brain by imaging mass spectrometry. J Lipid Res 50:1776-1788

2. Shimma S, Sugiura Y, Hayasaka T, Zaima N, Matsumoto M, Setou M (2008) Mass imaging and identification of biomolecules with MALDI-QIT-TOF-based system. Anal Chem 80:878-885

3. Khatib-Shahidi S, Andersson M, Herman JL, Gillespie TA, Caprioli RM (2006) Direct molecular analysis of whole-body animal tissue sections by imaging MALDI mass spectrometry. Anal Chem 78:6448-6456

4. Stoeckli M, Chaurand P, Hallahan DE, Caprioli RM (2001) Imaging mass spectrometry: a new technology for the analysis of protein expression in mammalian tissues. Nat Med 7:493-496

5. Groseclose MR, Andersson M, Hardesty WM, Caprioli RM (2007) Identification of proteins directly from tissue: in situ tryptic digestions coupled with imaging mass spectrometry. J Mass Spectrom 42:254-262

6. Edwards JL, Kennedy RT (2005) Metabolomic analysis of eukaryotic tissue and prokaryotes using negative mode MALDI time-of-flight mass spectrometry. Anal Chem 77:2201-2209

7. Hattori K, Kajimura M, Hishiki T, Nakanishi T, Kubo A, Nagahata Y, Ohmura M, Yachie-Kinoshita A, Matsuura T, Morikawa T et al (2010) Paradoxical ATP elevation in ischemic penumbra revealed by quantitative imaging mass spectrometry. Antioxid Redox Signal 13:1157-1167

8. Miura D, Fujimura Y, Yamato M, Hyodo F, Utsumi H, Tachibana H, Wariishi H (2010) Ultrahighly sensitive in situ metabolomic imaging for visualizing spatiotemporal metabolic behaviors. Anal Chem 82:9789-9796

9. Harada T, Yuba-Kubo A, Sugiura Y, Zaima N, Hayasaka T, GotoInoue N, Wakui M, Suematsu M, Takeshita K, Ogawa K et al (2009) Visualization of volatile substances in different organelles with an atmospheric-pressure mass microscope. Anal Chem 81:9153-9157

10. Cooks RG, Ouyang Z, Takats Z, Wiseman JM (2006) Detection Technologies. Ambient mass spectrometry. Science 311:1566-1570

11. Liu Q, Guo Z, He L (2007) Mass spectrometry imaging of small molecules using desorption/ionization on silicon. Anal Chem 79:3535-3541

12. Nemes P, Barton AA, Vertes A (2009) Three-dimensional imaging of metabolites in tissues under ambient conditions by laser ablation electrospray ionization mass spectrometry. Anal Chem 81:6668-6675

13. Li Y, Shrestha B, Vertes A (2008) Atmospheric pressure infrared MALDI imaging mass spectrometry for plant metabolomics. Anal Chem 80:407-420

14. Sroyraya M, Goto-Inoue N, Zaima N, Hayasaka T, Chansela P, Tanasawet S, Shrivas K, Sobhon P, Setou M (2010) Visualization of biomolecules in the eyestalk of the blue swimming crab, Portunus pelagicus, by imaging mass spectrometry using the atmosphericpressure mass microscope. Surf Interface Anal 42:1589-1592

15. Takizawa Y, Mizuta K, Hayasaka T, Nakanishi H, Okamura J, Mineta H, Setou M (2010) Specific localization of five phosphatidylcholine species in the cochlea by mass microscopy. Audiol Neurootol 16:315-322
16. Sun G, Yang K, Zhao Z, Guan S, Han X, Gross RW (2007) Shotgun metabolomics approach for the analysis of negatively charged water-soluble cellular metabolites from mouse heart tissue. Anal Chem 79:6629-6640

17. Hamada K, Monnai M, Kawai K, Nishime C, Kito C, Miyazaki N, Ohnishi Y, Nakamura M, Suemizu H (2008) Liver metastasis models of colon cancer for evaluation of drug efficacy using NOD/Shi-scid IL2R $\gamma^{\text {null }}$ (NOG) mice. Int J Oncol 32:153-159

18. Ito M, Hiramatsu H, Kobayashi K, Suzue K, Kawahata M, Hioki K, Ueyama Y, Koyanagi Y, Sugamura K, Tsuji K et al (2002) $\mathrm{NOD} / \mathrm{SCID} / \gamma \mathrm{c}^{\text {null }}$ mouse: an excellent recipient mouse model for engraftment of human cells. Blood 100:3175-3182

19. Handa K, Ohmura M, Nishime C, Hishiki T, Nagahata Y, Kawai K, Suemizu H, Nakamura M, Wakui M, Kitagawa Y et al (2010) Phosphorescence-assisted microvascular $\mathrm{O}_{2}$ measurements reveal alterations of oxygen demand in human metastatic colon cancer in the liver of superimmunodeficient NOG mice. Adv Exp Med Biol 662:423-429

20. Sakuragawa T, Hishiki T, Ueno Y, Ikeda S, Soga T, YachieKinoshita A, Kajimura M, Suematsu M (2010) Hypotaurine is an energy-saving hepatoprotective compound against ischemiareperfusion injury of the rat liver. J Clin Biochem Nutr 46:126134

21. Shintani T, Iwabuchi T, Soga T, Kato Y, Yamamoto T, Takano N, Hishiki T, Ueno Y, Ikeda S, Sakuragawa T et al (2009) Cystathionine $\beta$-synthase as a carbon monoxide-sensitive regulator of bile excretion. Hepatology 49:141-150

22. Soga T, Baran R, Suematsu M, Ueno Y, Ikeda S, Sakurakawa T, Kakazu Y, Ishikawa T, Robert M, Nishioka T et al (2006) Differential metabolomics reveals ophthalmic acid as an oxidative stress biomarker indicating hepatic glutathione consumption. J Biol Chem 281:16768-16776

23. Kinoshita A, Tsukada K, Soga T, Hishiki T, Ueno Y, Nakayama Y, Tomita M, Suematsu M (2007) Roles of hemoglobin allostery in hypoxia-induced metabolic alterations in erythrocytes: simulation and its verification by metabolome analysis. J Biol Chem 282:10731-10741

24. Accatino L, Simon FR (1976) Identification and characterization of a bile acid receptor in isolated liver surface membranes. J Clin Invest 57:496-508

25. Stahl E, Arnesjo B (1972) Taurocholate metabolism in man. Scand J Gastroenterol 7:559-566

26. Pacifici GM, Rane A (1982) Distribution of UDP-glucuronyltransferase in different human foetal tissues. Br J Clin Pharmacol 13:732-735

27. Suematsu M, Kato S, Ishii H, Asako H, Yanagisawa T, Suzuki H, Oshio C, Tsuchiya M (1991) Intralobular heterogeneity of carbon tetrachloride-induced oxidative stress in perfused rat liver visualized by digital imaging fluorescence microscopy. Lab Invest 64:167-173

28. Ishimoto T, Nagano O, Yae T, Tamada M, Motohara T, Oshima H, Oshima M, Ikeda T, Yagi H, Masuko T, et al. (2011) CD44 variant regulates redox status in cancer cells by stabilizing the $\mathrm{xCT}$ subunit of system xc- and thereby promotes tumor growth. Cancer Cell 19:387-400

29. Moscow JA, Fairchild CR, Madden MJ, Ransom DT, Wieand HS, O'Brien EE, Poplack DG, Cossman J, Myers CE, Cowan KH (1989) Expression of anionic glutathione-S-transferase and Pglycoprotein genes in human tissues and tumors. Cancer Res 49:1422-1428

30. Dang DT, Chen F, Kohli M, Rago C, Cummins JM, Dang LH (2005) Glutathione S-transferase pil promotes tumorigenicity in HCT116 human colon cancer cells. Cancer Res 65:9485-9494 